Article

\title{
In Vivo Assessment of Bone Enhancement in the Case of 3D-Printed Implants Functionalized with Lithium-Doped Biological-Derived Hydroxyapatite Coatings: A Preliminary Study on Rabbits
}

\author{
Liviu Duta ${ }^{1, * \mathbb{C}}$, Johny Neamtu ${ }^{2}$, Razvan P. Melinte ${ }^{2}$, Oana A. Zureigat ${ }^{2}$, \\ Gianina Popescu-Pelin ${ }^{1}$, Diana Chioibasu ${ }^{3,4}$, Faik N. Oktar ${ }^{5,6}$ and Andrei C. Popescu ${ }^{3, *}$ \\ 1 National Institute for Lasers, Plasma and Radiation Physics, 077125 Magurele, Romania; \\ gianina.popescu@inflpr.ro \\ 2 Faculty of Pharmacy, University of Medicine \& Pharmacy, 200349 Craiova, Romania; \\ johny.neamtu@umfcv.ro (J.N.); razvan.melinte@gmail.com (R.P.M.); oana.toba@yahoo.com (O.A.Z.) \\ 3 Center for Advanced Laser Technologies (CETAL), National Institute for Lasers, Plasma and Radiation \\ Physics, 077125 Magurele, Romania; diana.chioibasu@inflpr.ro \\ 4 Faculty of Applied Sciences, Politehnica University of Bucharest, 060042 Bucharest, Romania \\ 5 Department of Bioengineering, Faculty of Engineering, Marmara University, Istanbul 34722, Turkey; \\ foktar@marmara.edu.tr \\ 6 Center of Nanotechnology \& Biomaterials Application \& Research, Marmara University, \\ Istanbul 34722, Turkey \\ * Correspondence: liviu.duta@inflpr.ro (L.D.); andrei.popescu@inflpr.ro (A.C.P.); \\ Tel.: +40-21-457-4550 (ext. 2023) (L.D.); +40-21-457-4550 (ext. 2423) (A.C.P.)
}

Received: 15 September 2020; Accepted: 15 October 2020; Published: 17 October 2020

check for updates

\begin{abstract}
We report on biological-derived hydroxyapatite (HA, of animal bone origin) doped with lithium carbonate (Li-C) and phosphate (Li-P) coatings synthesized by pulsed laser deposition (PLD) onto Ti6Al4V implants, fabricated by the additive manufacturing (AM) technique. After being previously validated by in vitro cytotoxicity tests, the Li-C and Li-P coatings synthesized onto 3D Ti implants were preliminarily investigated in vivo, by insertion into rabbits' femoral condyles. The in vivo experimental model for testing the extraction force of $3 \mathrm{D}$ metallic implants was used for this study. After four and nine weeks of implantation, all structures were mechanically removed from bones, by tensile pull-out tests, and coatings' surfaces were investigated by scanning electron microscopy. The inferred values of the extraction force corresponding to functionalized 3D implants were compared with controls. The obtained results demonstrated significant and highly significant improvement of functionalized implants' attachment to bone ( $p$-values $\leq 0.05$ and $\leq 0.00001$ ), with respect to controls. The correct placement and a good integration of all 3D-printed Ti implants into the surrounding bone was demonstrated by performing computed tomography scans. This is the first report in the dedicated literature on the in vivo assessment of Li-C and Li-P coatings synthesized by PLD onto Ti implants fabricated by the AM technique. Their improved mechanical characteristics, along with a low fabrication cost from natural, sustainable resources, should recommend lithium-doped biological-derived materials as viable substitutes of synthetic HA for the fabrication of a new generation of metallic implant coatings.
\end{abstract}

Keywords: biological-derived hydroxyapatite coatings; lithium doping; food industrial by-products; in vivo extraction force; pulsed laser deposition; 3D printing 


\section{Introduction}

The increase of life expectancy and the enhanced frequency of injuries and diseases are considered the most important causes for the escalating demand for dental and orthopedic devices. In this respect, the surface functionalization of implants with highly performant bioactive materials is currently of interest and necessary both for the prevention of failure and the prolongation of the bone implants' life. Thus, good examples are calcium phosphates (CaPs) and bioglasses [1-4], which are the most used bioceramic materials in medicine, in particular in orthopedics and dentistry [5,6], as coatings for various metallic implants [7]. From the first class, a special focus was put on hydroxyapatite (HA), $\mathrm{Ca}_{10}\left(\mathrm{PO}_{4}\right)_{6}(\mathrm{OH})_{2}[3,4,7]$. Due to their role as scaffolds for osteogenic differentiation [8], the ability to form strong bonds with the host bone tissues [9], and their excellent capacity to stimulate and accelerate the formation of new bone tissue around implants [10-12], HA ceramics are frequently utilized in bone grafting and dental devices as bone substitutes, either in their simple form, or in conjunction with other different biomaterials $[13,14]$. One should note that, if for the healthy patients, the osseointegration rate takes place at a reasonable speed, in the case of older patients, or those presenting critical medical conditions from the systemic point of view (in particular HIV positive patients that reported an increase in early failures of dental implants $[15,16])$, this process could be improved by applying HA-based coatings on to the implants' surfaces.

Despite its excellent bone-regeneration properties, HA is brittle in bulk [17] and characterized by poor mechanical properties. Usually, an implant can be manufactured from Ti or its medical-grade alloys. In this respect, additive manufacturing (AM) is a technology that allows for cost-effective and rapid production of complex three dimensional (3D) metallic parts and is gaining nowadays increased attention in the field of personalized medicine $[18,19]$. In contrast to their excellent mechanical properties, Ti implants elicit low osseointegration rates. To overpass this shortcoming, HA can be applied as a coating onto the surface of Ti implants, to significantly improve the overall performances of the structures, by successfully combining the excellent bioactivity of the ceramic with the mechanical advantages of the metallic substrates $[17,20,21]$. To enhance these properties even more, HA doping with various concentrations of therapeutic ions is envisaged $[4,7,22,23]$.

Currently, huge interest is focused on finding appropriate deposition techniques to modify the surface of metallic implants, by functionalization with coatings, proteins and/or drugs that could favor the enhancement of cellular adhesion, thus leading to the acceleration of the osseointegration time [24-27]. In the field of thin-film growth, pulsed laser deposition (PLD) has been established in the past 15-20 years as one of the most popular and efficient methods for the deposition of a wide spectrum of materials, especially onto substrates with complex geometrical shapes, including implants [28]. Moreover, when CaPs of animal origin are involved, the PLD technique could be one of the most suitable choices to synthesize this type of coating. If the synthetic HA has a complex stoichiometry, with a large number of atoms and functional groups difficult to be transferred in the form of thin layers by physical vapor deposition techniques, biological apatites become even more difficult to transfer due to the supplemental presence of functional groups and substitutional ions, which further complicate their stoichiometry and structure [29]. Nevertheless, the PLD method is well-known for its ability to stoichiometrically transfer very complex molecules (due to the high ablation rate which causes all elements to evaporate at the same time [30]) and this advantage should place it among the main candidates for the successful transfer of such complex materials. Moreover, with the major drawback of this deposition technique (i.e., being capable of coating only small-area substrates, as the plasma plume diameter is restricted by the low spot size of the laser beam) being overcome by the implementation of laser scanning units in commercially, widely available PLD equipment, the path towards large-scale application is thus opened [31,32].

It is a documented fact that in vitro tests can play an important role in the evaluation of a material by providing useful information about its potential behavior in a biological environment. To better understand the complex processes that may occur in a living system and to provide the most accurate data for the complete confirmation of the performance of a biomaterial designed for clinical trials, 
in vivo tests in animal models are extremely important and should, therefore, follow thorough in vitro confirmation. Accordingly, after previous validation of both in vitro cytocompatibility [33,34] and antimicrobial activity [35] of biological HA (BHA) doped with lithium carbonate (further denoted as Li-C) and phosphate (further denoted as Li-P) coatings, a leap forward in our research was performed by investigating their preliminary bone bonding and bone bonding strength characteristics in vivo in an animal model. Thus, the aim of this preliminary study was both to (i) demonstrate superior bone-implant interactions in the case of PLD functionalized implants as compared to simple, uncoated ones (Ti controls), and (ii) verify the hypothesis that higher values of the extraction force are obtained in the case of implants extracted after longer periods of implantation time. It should be stressed that there are reports in the literature on the in vivo testing of CaPs (especially HA), but, to the best of our knowledge, the novelty of the current work resides both in the additive manufacturing (AM) technique used to fabricate the implantable medical devices, and the fact that this is the first preliminary study to address the in vivo mechanical behavior of these 3D printed implants functionalized by PLD with biological HA, doped with Li-C and Li-P coatings.

\section{Materials and Methods}

\subsection{Printing of Metallic Implants}

The laser melting deposition (LMD) technique was used for the manufacturing of three-dimensional (3D) metallic implants. A Ti6Al4V powder (with the particle size of $<90 \mu \mathrm{m}$ ), further denoted as control, was used as precursor material. Implants were in " $\mathrm{T}$ "-shape (Figure 1a,b) and their dimensions are displayed in Figure 1a. It should be mentioned that, by using the LMD technique, the fabrication costs and manufacturing time of 3D metallic implants were significantly reduced.

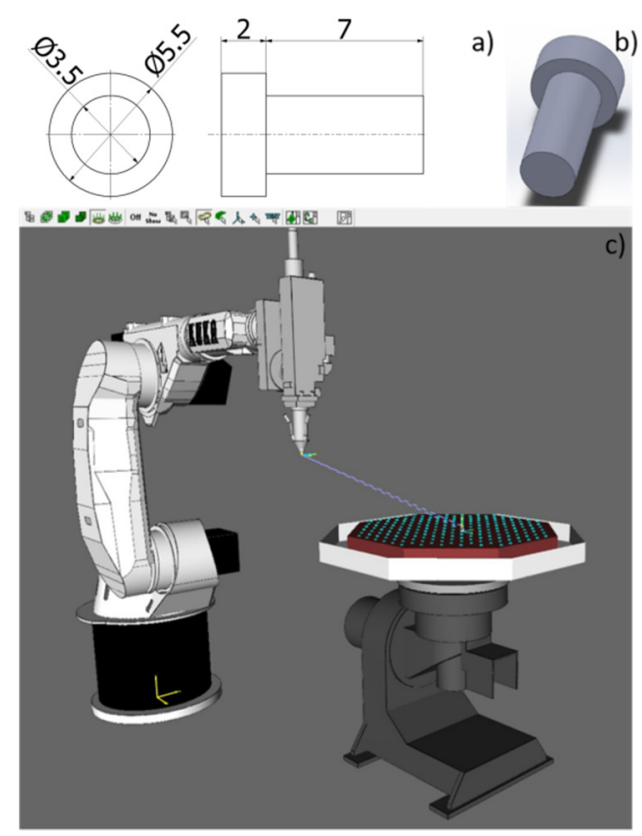

Figure 1. (a) The technical drawing of the 3D metallic implant; (b) the 3D drawing of the implant; (c) the TruTops Cell ${ }^{\circledR}$ graphical software which generates the movement codes for the robotic arm.

First, incipient bulk prisms of $(10 \times 5 \times 1) \mathrm{cm}^{3}$ were $3 \mathrm{D}$ printed using several experimental conditions, to find the optimal parameters in view of obtaining defect-free samples. Thus, by optimization of the laser and scanning parameters, compact structures without cracks or pores and with excellent compositional uniformity were obtained. The laser beam (supplied through an optical fiber) used for melting the powder 
was generated by an Yb:YAG disk source $(\lambda=1030 \mathrm{~nm}$, continuous wave), model TruDisk 3001 (Trumpf, Ditzingen, Germany). The surface focused spot size had a diameter of $\sim 0.6 \mathrm{~mm}$.

An automated powder feeder (Trumpf, Ditzingen, Germany), connected through hoses (having $6 \mathrm{~mm}$ diameters) to a nozzle with three flow channels, that was mounted on a robotic system TruLaser Robot 5020 (Trumpf, Ditzingen, Germany), made of a Kr30HA robot (Kuka, Augsburg, Germany), with six movement axes, was used for the powder delivery. The powder was sent to the robot through a delivery system provided with turntables. A three-beam particle flow nozzle was used and aligned so that the beams could be coherent in a single point, congruent with the laser beam spot. The Ti implants were fabricated under Ar ambient.

The 2D technical drawing of the 3D metallic implants (Figure 1a) was generated in the graphical engineering software Solid Works ${ }^{\circledR}$ (Dassault Systems, Vélizy-Villacoublay, France), then translated to the 3D space (Figure 1b), and subsequently imported in TruTops Cell ${ }^{\circledR}$ (Trumpf, Ditzingen, Germany) (Figure 1c), a movement code generator for the robotic arm.

The design of the trajectory followed by the laser beam for structure growth proved to be essential to obtain samples that respect both the appearance and dimensions of the technical drawings. The separation distance of contours on the z-axis, established by tracing a calibration curve, was of $0.5 \mathrm{~mm}$. All trajectories executed by the robotic arm were extremely important to obtain a homogeneous structure, free of discontinuities.

Three-dimensional metallic implants were fabricated onto a Ti substrate with a thickness of $10 \mathrm{~mm}$, after which they were removed from it by a disk cutting machine model Brillant 200 (ATM, Mammelzen, Germany).

\subsection{Pulsed Laser Deposition (PLD) Experiments}

\subsubsection{Powders}

Biological-derived hydroxyapatite (BHA) powders were obtained from the cortical part of bovine femoral bones, according to the protocol described in [29]. Concisely, heads of femoral bones were cut-off and shafts were further processed. The bone marrows were then extracted and the unwanted soft tissue residues or macroscopic adhering impurities and substances were gently removed from shafts, which were sliced, cleaned and washed with distilled water and deproteinized for 14 days in an alkali media of $1 \%$ sodium hypochlorite. The elimination of any potential hazardous contaminants was achieved by a calcination process (at $850^{\circ} \mathrm{C}$, for $4 \mathrm{~h}$, in air) of the resulting dry bone fragments [36,37]. Prior to undergoing ball-milling to fine powders, the calcined bone specimens were crushed with a mortar and pestle. It should be emphasized that, for the fabrication of BHA powders, European Union (EU) regulations [38] and ISO 22442-1 [39] were followed. Batches of BHA fine powders were admixed with 1 wt.\% of Li-C and Li-P (Sigma-Aldrich GmbH, St. Louis, MO, USA).

\subsubsection{Target Preparation}

The as-obtained Li-C and Li-P mixed powders were pressed at $\sim 6 \mathrm{MPa}$ in a $20 \mathrm{~mm}$ diameter mold. The resulting pellets were thermally treated in air, using an oven, for $4 \mathrm{~h}$, at $700{ }^{\circ} \mathrm{C}$. A heating rate of $20^{\circ} \mathrm{C} / \mathrm{min}$ and a cooling ramp of $5^{\circ} \mathrm{C} / \mathrm{min}$ were applied. Following this protocol, the fabrication of hard and compact targets was carried out.

\subsubsection{Coating Fabrication}

PLD experiments were conducted inside a stainless-steel deposition chamber, in an ambient water vapor pressure of $50 \mathrm{~Pa}$. The target-to-substrate separation distance was of $5 \mathrm{~cm}$. Coatings were synthesized using a $\mathrm{KrF}^{*}$ excimer laser source (COMPexPro 205, Coherent, Santa Clara, CA, USA, $\lambda=248 \mathrm{~nm}, \tau_{\mathrm{FWHM}} \leq 25 \mathrm{~ns}$ ). The incident laser fluence was set at $3.5 \mathrm{~J} / \mathrm{cm}^{2}$ (with a corresponding pulse energy of $360 \mathrm{~mJ}$ ). The laser beam was incident at $45^{\circ}$ on the target surface. For the growth of one film, 15000 consecutive laser pulses were applied. During the multi-pulse laser irradiation, the target 
was continuously rotated with $0.3 \mathrm{~Hz}$ and translated along two orthogonal axes, to avoid piercing and to obtain unidirectional plasma.

Prior to introduction into the deposition chamber, all substrates were successively cleaned following a three-step protocol [34]. In addition, to avoid any possible micro-contamination, targets were submitted to a "cleaning" process with 1000 laser pulses. To collect the flux of expulsed micro-impurities, a shutter was interposed between the target and the 3D metallic substrates.

To ensure the complete deposition of the 3D Ti implants, a rotating stainless-steel support flange and four clamping pins [40] were used. During depositions, the substrates' temperature was kept constant at $(500 \pm 5 \%){ }^{\circ} \mathrm{C}$, using a heater connected to a PID-EXCEL temperature controller (EXCEL Instruments, Mumbai, India). A heating rate of $25^{\circ} \mathrm{C} / \mathrm{min}$ and a cooling ramp of $10^{\circ} \mathrm{C} / \mathrm{min}$ were applied.

\subsubsection{Thermal Treatments}

Both the temperature used for heating the substrates during experiments and post-deposition thermal treatments, were demonstrated to have an important influence on the coatings' characteristics and, in particular, on their composition and stoichiometry. Therefore, all synthesized structures were submitted to post-deposition thermal-treatments in water-vapors enriched atmosphere. A removable flange, positioned on the heater so as to ensure an optimal contact with the substrates, was used.

\subsection{Animals and Surgical Experimental Protocol}

The animal spectrum generally used to test CaP coatings synthesized by the PLD technique is limited to rats, mini-pigs, dogs, goats, sheep and rabbits [17]. Both the advantages and disadvantages of using different animal models have been widely discussed in the study reported by Lu et al. [41]. It should be emphasized that, the rabbit represents one of the most used animals for medical studies [42], due to its size and ease of handling. Moreover, its skeleton reaches maturity in a rather short period of time (approximatively six months) [43].

For the in vivo experiments reported in this study, that took place in the biobase of the University of Medicine and Pharmacy (UMF), Craiova, Romania, a total of 26 skeletally-matured New Zealand White rabbits, aged six months and weighing between 3 and $3.5 \mathrm{~kg}$ were used. One should note that, animals were weighed at the beginning of the study and before sacrifice, and no significant weight changes were observed. All animals were free of disease. They were housed in individual plastic cages, in a climate-controlled environment at $22{ }^{\circ} \mathrm{C}, 45 \%$ humidity, and 12-h alternating light-dark cycles. During the experimental period, free access to a standard laboratory diet and tap water were provided. All animals were acclimatized for 10 days before use in this study.

Before surgery, the control and functionalized 3D Ti implants were sterilized by autoclaving (at $120^{\circ} \mathrm{C}$, for $1 \mathrm{~h}$ ).

The 26 rabbits were randomly assigned to two groups ( $n=13$ each). The 3D metallic implants were introduced into the femoral condyles [44] (two implants in each rabbit), using the following sequence: at the level of the right femur, the 3D Ti implants functionalized with Li-C and/or Li-P coatings, and at the level of the left femur, the simple Ti implants (controls).

Antibiotic prophylaxis was performed pre-operatively, by intramuscular administration of a cephalosporin (sulfate diluted in physiological serum, at a dose of $40 \mathrm{mg} / \mathrm{kg}$ body). The antibiotic treatment continued for up to seven days (two doses/day).

All surgeries were performed under general anesthesia, following a three-step procedure: (i) sedation by subcutaneous administration of fentanyl $(0.1 \mathrm{~mL} / \mathrm{Kg})$ and midazolam $(2 \mathrm{mg} / \mathrm{Kg})$, (ii) maintenance (during the surgical act) of the anesthesia by administration of diluted fentanyl with physiological serum ( $1 \mathrm{~mL}$ fentanyl to $9 \mathrm{~mL}$ physiological serum), and (iii) injection at the incision site (under sterile conditions), of $1 \%$ xylin $(5 \mathrm{~mL})$, as an adjuvant.

At the beginning of the surgical procedure, the incision site was shaved and washed well with water and soap, and disinfected with betadine solution, followed by covering the animal with sterile 
overlays. An incision of $\sim 3 \mathrm{~cm}$ was performed on the lateral face of the distal femoral epiphysis. This interested the epidermis, the dermis, and the facial layers. The muscle present at this level was dissociated and the periosteum was incised longitudinally, exposing the bony lateral face of the distal femoral epiphysis.

Using a dental burr (Stryker Core Reamer), with adjustable rotational drill speed, under continuous saline irrigation, to which burr drills (Osstem surgical kit for dental implants, model TS III SA) with progressive diameter were attached, an opening hole was drilled through the cortical bone. Later, this hole was enlarged progressively (to avoid a possible cortical fracture), to a diameter slightly inferior to the implant's dimensions. Into the bone socket thus created, the 3D metallic implant was inserted in a press-fit manner, ensuring both complete coverage of its surface and stability. The implant was then carefully covered and protected by soft tissues and the muscle fascia and epidermis were closed with sutures (Figure 2).
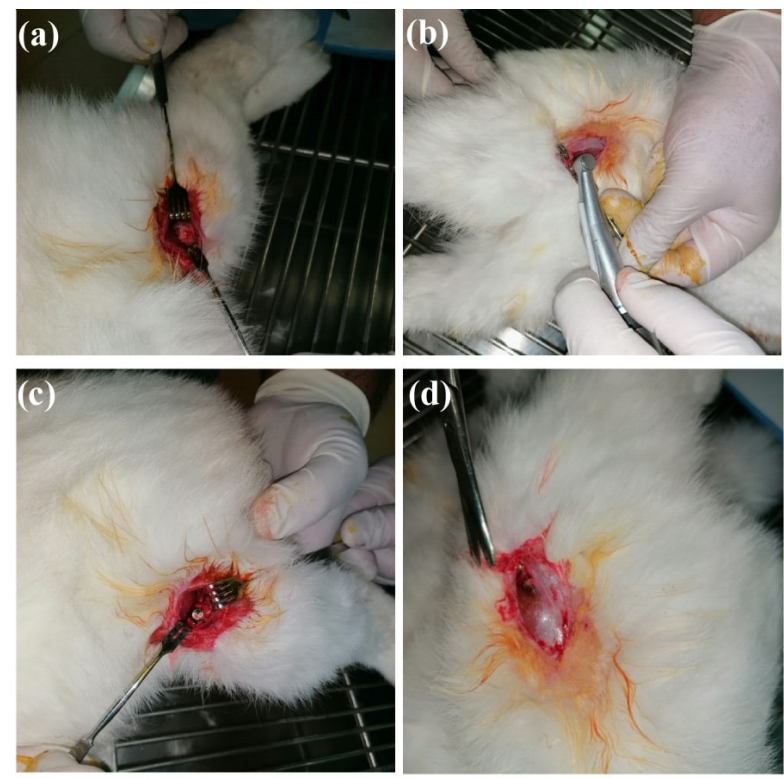

Figure 2. Surgical steps for the correct positioning of the 3D Ti implant: (a) exposure of lateral side of the distal femur, with visualization of the cortical bone; (b) opening hole drilled through the cortical bone using a dental burr; (c) insertion of the implant into the bone socket in a press-fit manner; (d) cover and protection of the implant by soft tissues and closing of the wound.

Immediate postoperative radiological examinations by computed tomography (CT) were performed both to confirm the correct position of the implants and to rule out any bone fractures or other possible surgical complications.

After the surgery, all animals were allowed to move freely in their cages, being monitored for general health conditions, on a daily basis. The operative wound was carefully checked and bandaged until complete healing. At the end of the implantation procedures, all rabbits were permitted free access to normal pellet food and water. No post-operative complications were reported.

The surgical protocol used for introducing the 3D metallic implants into the rabbits' femoral condyles was approved by the "Committee of Ethics and Academic and Scientific Deontology" at the UMF in Craiova, Romania (document no. 135/20.12.2019), and the entire experimental process and the surgical technique complied with regulations and precautions of the EU Council Directive of 22 September 2010, regarding the care and use of laboratory animals for scientific purposes (2010/63/EU). 


\subsection{Characterization of Control and Functionalized Three-Dimensional (3D) Ti Implants}

\subsubsection{Computed Tomography}

Investigations were performed using a Siemens CT scanner, operated at $130 \mathrm{kV}, 90 \mathrm{~mA}, 0.5 \mathrm{~mm}$ section thickness and $0.3 \mathrm{~mm}$ section increment. The integration and total scan times were of $0.5 \mathrm{~s}$ and $2 \mathrm{~min}$, respectively. For the evaluation of bone density, the Onis 2.3.5 software was used, and the inferred values were expressed on the Hounsfield tissue density scale (HU units). The area on the CT sections in which the tissue density was evaluated (also known as the region of interest), was always selected from the same region of the implant, for each performed measurement.

\subsubsection{Mechanical Testing}

Four weeks after the insertion of the 3D metallic implants, the rabbits were anesthetized (following the same protocol described above), and euthanized by intracardiac injection, using an overdose of sodium pentobarbital $(100 \mathrm{mg} / \mathrm{kg})$.

The anterior incision was resumed and the distal femoral epiphysis, containing the implant after disarticulation and proximal sectioning of the femoral diaphysis (Figure 3), was harvested for evaluation. After careful dissection and cleaning of any adherent soft tissues, the bone tissue-implant block specimens were obtained and immediately fixed in $5 \%$ buffered formaldehyde solution, prior to being subjected to mechanical tests (within $1 \mathrm{~h}$ of euthanasia).
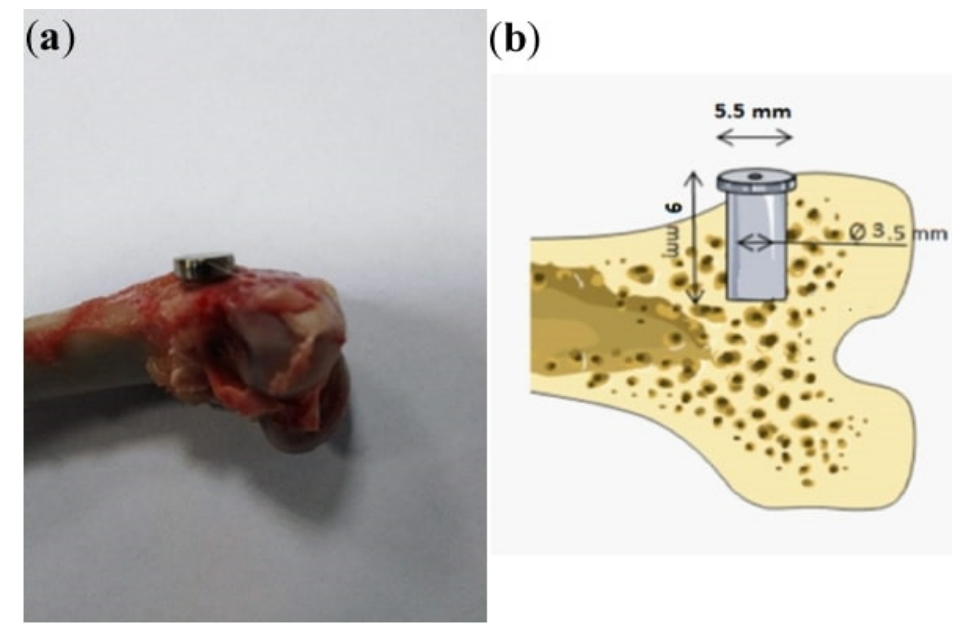

Figure 3. (a) Photograph of the extracted bone; (b) schematic representation of the implant site.

The quantification of the in vivo interfacial extraction force of bone implants represents a biological challenge. One should note that, the analysis of the phenomena that occur at the bone tissue-implant interface is a complex procedure, which requires the use of experimental cutting and grinding techniques, which are quite complicated and expensive. To avoid these shortcomings, the experimental model of in vivo testing of the extraction force of 3D metallic implants was, therefore, applied in this study.

It is important to mention that, the retention between the implant and the bone was evaluated before euthanasia could influence the results (within the first $4 \mathrm{~h}$ after euthanasia).

The measurement of the implants extraction force was performed by a tensile traction machine (model WDW, Time Group), which measures both the force and the elongation, and can operate with a maximum force of $5000 \mathrm{~N}$. All tests were performed with a traction speed of $1 \mathrm{~mm} / \mathrm{min}$. The implant was positioned into the traction machine support by means of a hexagonal adapter attached with an adhesive on the exterior side of the 3D Ti implant (Figure 4). One should take into account that, when fixing the femur and the implant, the position of the 3D metallic implant should be aligned with 
the traction direction. Typical load-displacement curves were recorded (data not shown here), and the failure load was defined as the peak load value of the load-displacement curve.
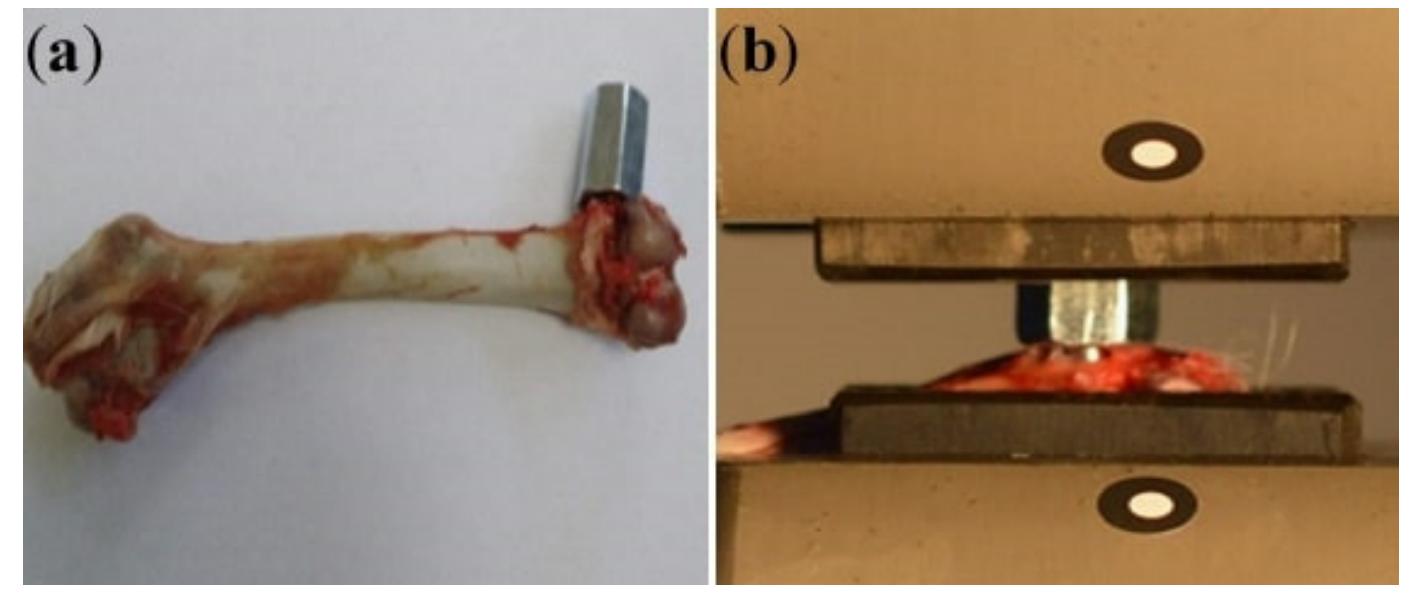

Figure 4. Tensile pull-out measurement: photographs of (a) the hexagonal adaptor attached to the 3D $\mathrm{Ti}$ implant, and (b) both the femur and the adaptor attached to the 3D metallic implant, in the traction machine support.

\subsubsection{Scanning Electron Microscopy (SEM)}

The morphological analysis of the 3D metallic implants' surfaces, following their extraction from the rabbits' femoral condyles, was performed. Therefore, to identify the adherent bone components, surfaces of both control and functionalized 3D Ti implants were examined by scanning electron microscopy (SEM). Using the analysis of backscattering electrons, along with the ImageJ free software (version 1.46r), one could estimate the adherence ratio of the bone tissue remaining on the implant's surface after its extraction from the rabbits' femoral condyle. This parameter is defined as the ratio between the estimated area of the remaining bone tissue and the area of the 3D implant (the area of a circle with a diameter of $3.5 \mathrm{~mm})$.

A SEM SU5000 (Hitachi, Tokyo, Japan), with a resolution of $1.2 \mathrm{~nm}$ and an acceleration voltage of $25 \mathrm{kV}$, was used for these investigations. For comparison reasons only, SEM micrographs of control Ti and Li-C and Li-P structures before surgery are also presented.

\subsection{Statistical Analysis}

Experiments were carried out both in decuplicate $(n=10$, for extractions performed at four weeks), and triplicate ( $n=3$, for extractions performed at nine weeks), to achieve statistical significance. The unpaired Student's $t$-test was used to determine the level of significance, and $p$-values $\leq 0.05$, $\leq 0.0001$ and $\leq 0.00001$ were considered to be significant and highly significant, respectively.

\section{Results}

\subsection{Clinical Observations Following Implantation}

Four weeks after the implantation procedure, the performed CT scans demonstrated the correct placement and a good integration of all implants into the surrounding bone (Figure 5). 


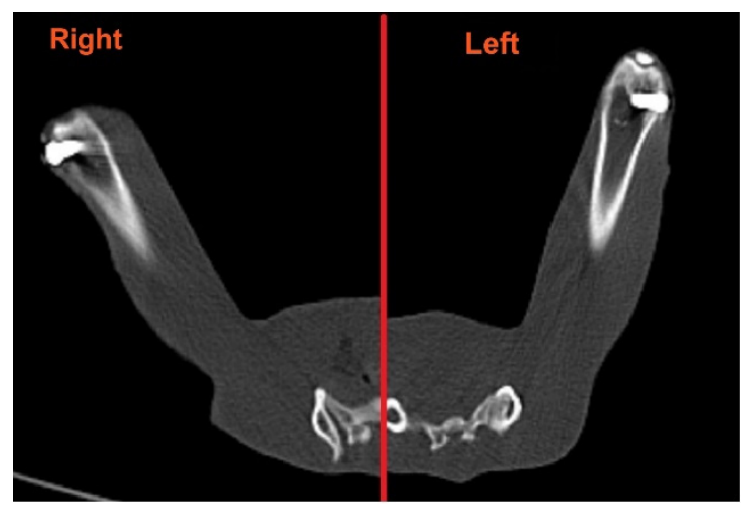

Figure 5. Lateral computed tomography images showing the positioning of the 3D metallic implants (right foot-implant functionalized with Li-C coating, left foot—control Ti implant), at 4 weeks after surgery.

The results of the osseous density measurements at the implant-bone interface, for control and functionalized (with Li-C and Li-P coatings) 3D Ti implants, are summarized in Tables 1 and 2.

Table 1. Osseous density (mean \pm standard deviation, SD) inferred at the implant-bone interface for control and functionalized (with Li-C coatings) 3D Ti implants.

\begin{tabular}{ccc}
\hline \multirow{2}{*}{ Implant Type } & \multicolumn{2}{c}{ Osseous Density (Mean \pm SD) [HU] } \\
\cline { 2 - 3 } & $\mathbf{4}$ Weeks & $\mathbf{9}$ Weeks \\
\hline Control (Ti) & $811 \pm 21$ & $850 \pm 57$ \\
Li-C & $1068 \pm 70$ & $1156 \pm 40$ \\
\hline
\end{tabular}

Table 2. Osseous density (mean $\pm \mathrm{SD}$ ) inferred at the implant-bone interface for control and functionalized (with Li-P coatings) 3D Ti implants.

\begin{tabular}{ccc}
\hline \multirow{2}{*}{ Implant Type } & \multicolumn{2}{c}{ Osseous Density (Mean \pm SD) [HU] } \\
\cline { 2 - 3 } & $\mathbf{4}$ Weeks & $\mathbf{9}$ Weeks \\
\hline Control (Ti) & $818 \pm 36$ & $855 \pm 61$ \\
Li-P & $1053 \pm 40$ & $1172 \pm 28$ \\
\hline
\end{tabular}

For all investigated 3D Ti implants, CT scans revealed at the implant-bone interface the presence of the peripheral osteosclerosis, and no inflammatory process of the soft tissues.

The results presented in Tables 1 and 2 point out to an increase of the osseous density, for both investigated time periods. Therefore, the bone density values corresponding to the 3D Ti implants functionalized with both Li-C and Li-P coatings, measured at 9 weeks, were $\sim 1.2$ times higher than those inferred at 4 weeks after surgery, respectively. At 4 weeks after surgery, both functionalized 3D Ti implants (with Li-C and Li-P coatings) showed bone density values 1.3 times higher than those obtained in the case of control 3D Ti implants. Moreover, at 9 weeks, the density values inferred in the case of functionalized 3D Ti implants were 1.4 times higher as compared to control ones.

One should also stress upon that, all rabbits were able to walk normally within $6 \mathrm{~h}$ after the implantation surgery. Moreover, no macroscopic signs of infection or adverse reactions were observed, and none of the rabbits died or suffered a bone fracture during this study.

\subsection{Mechanical Testing}

Mechanical tests were used to evaluate the quality of the implants' osseointegration. It is important to note that no implant showed surface alteration or disruption. 
In Figures 6 and 7 are represented the characteristic values of the load to failure (detachment force, $\left.F_{\max }\right)$ of implants under tensile pull-out testing, inferred for control and functionalized 3D metallic implants, at 4 and 9 weeks after surgery, respectively.
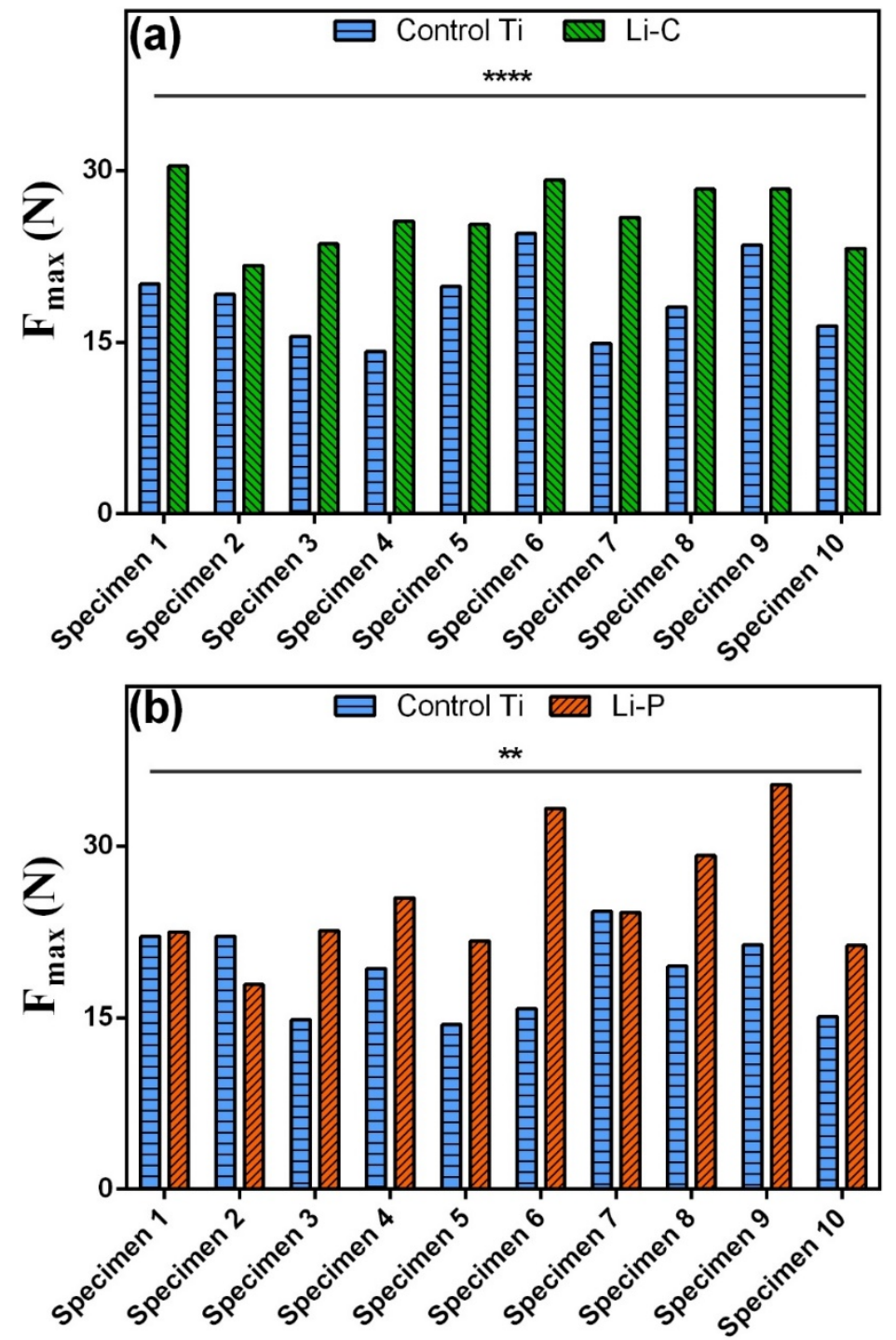

Figure 6. Detachment force, $F_{\max }$, of implants $(n=10)$ under tensile pull-out testing, inferred in the case of control 3D Ti implants (marked in blue color) and of those functionalized with (a) Li-C (marked in green color) and (b) Li-P (marked in orange color) coatings, at 4 weeks after surgery. ${ }^{* * *}$ Represents highly significant differences $(p \leq 0.0001)$. ${ }^{* *}$ Represents significant differences $(p \leq 0.01)$. 

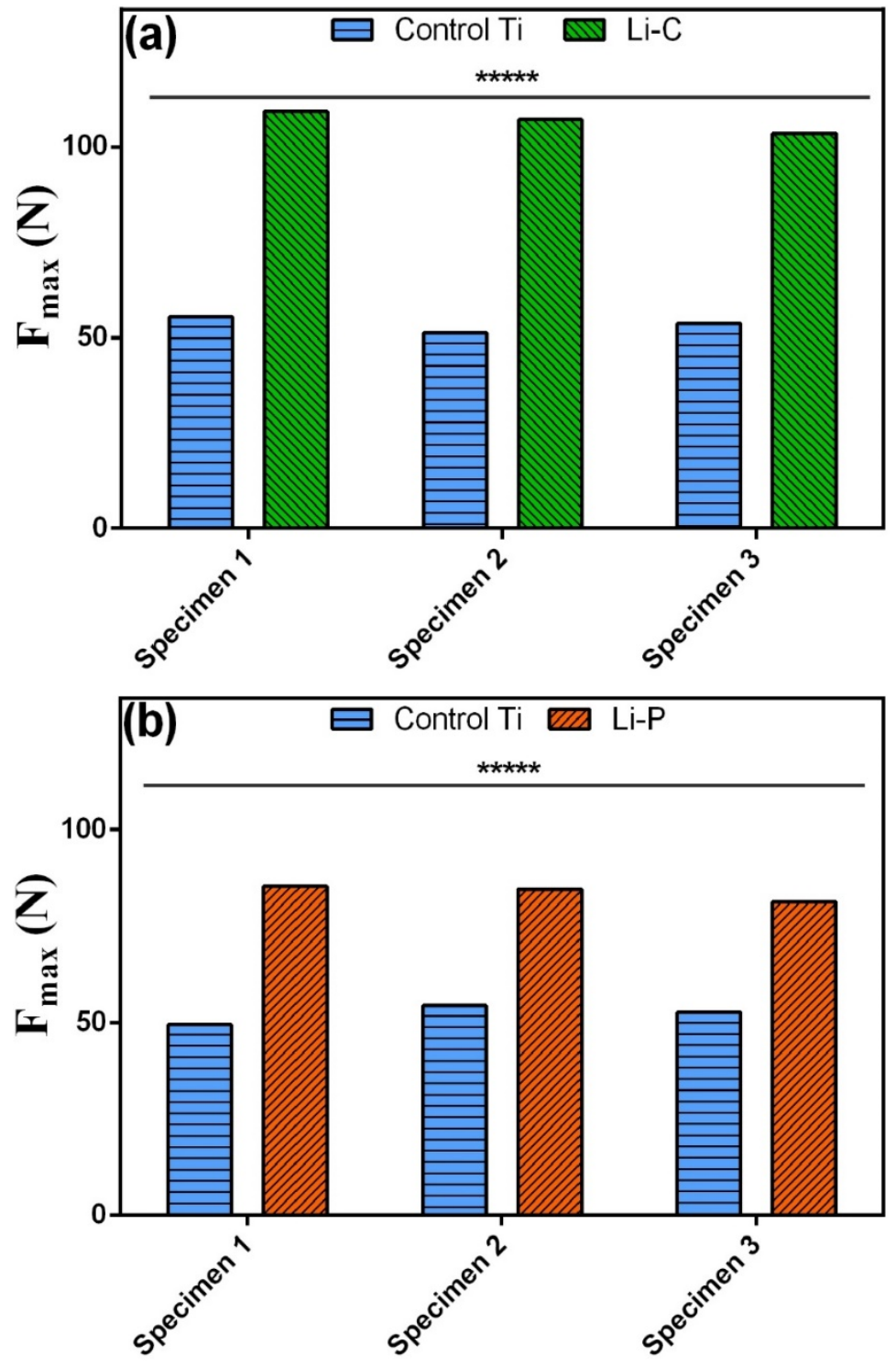

Figure 7. Detachment force, $F_{\max }$, of implants $(n=3)$ under tensile pull-out testing, inferred in the case of control 3D Ti implants (marked in blue color) and of those functionalized with (a) Li-C (marked in green color) and (b) Li-P (marked in orange color) coatings, at 9 weeks after surgery. ${ }^{* * * *}$ Represents highly significant differences $(p \leq 0.00001)$.

In the case of the extraction tests performed at 4 weeks (Figure $6 a, b)$, the obtained mean detachment force values demonstrated (i) a highly significant difference between $18.6 \mathrm{~N}( \pm 3.5)$, for the first control Ti group $(n=10)$, and $26.2 \mathrm{~N}( \pm 2.9)$, for the Li-C test group $(n=10)(p=0.00006)$ (Figure 6a), and (ii) a significant difference between $18.8 \mathrm{~N}( \pm 3.5)$, for the second control Ti group $(n=10)$, and $25.4 \mathrm{~N}( \pm 5.6)$, for the Li-P test group $(n=10)(p=0.007)$ (Figure 6b).

When referring to the extractions performed at longer time periods, i.e., 9 weeks from surgery (Figure 7a,b), the inferred mean detachment force values indicated highly significant differences for both investigated cases: between $53.5 \mathrm{~N}( \pm 2.2)$, for the first control Ti group $(n=3)$, and 106.6 $\mathrm{N}( \pm 2.9)$, for the Li-C test group $(n=3)(p=0.00003)$ (Figure 7a), and between $52.1 \mathrm{~N}( \pm 2.5)$, for the second control Ti group $(n=3)$, and $83.7 \mathrm{~N}( \pm 2.1)$, for the Li-P test group $(n=3)(p=0.00009)$ (Figure 7b).

One can observe that, the failure loads of 3D Ti implants functionalized with both Li-C and Li-P coatings measured at 9 weeks were (3.4-5) and (2.3-4.8) times higher than those inferred at 4 weeks after surgery, respectively (Figures 6 and 7). It should be stressed here that, a similar trend was also 
observed by Yan et al., in the case of strontium-containing HA coatings [45]. Moreover, at 4 weeks after surgery, the 3D Ti implants functionalized with Li-C and Li-P coatings showed a bone attachment strength of about (1.1-1.8) and (1.1-2.1) times stronger than that corresponding to the control 3D Ti implants, respectively. After 9 weeks of implantation, the inferred values of the attachment force were about (1.9-2.1) and (1.5-1.7) times higher than controls, in the case of Li-C and Li-P samples, respectively. Therefore, one could indicate that both the PLD surface functionalization of 3D Ti implants and a longer implantation time period could positively influence the overall bone bonding strength characteristics of the investigated medical devices. With this result, the aim of this study was attained.

\subsection{SEM}

Immediately after deposition, the surfaces of control and functionalized 3D Ti implants were examined by SEM, under two different magnifications (1000 $\times$ and $10,000 \times$, respectively). Typical SEM micrographs of control 3D Ti implants (Figure 8) show irregular morphologies which are due to the micro-machining preparation process only. The deposition of $\mathrm{Li}-\mathrm{C}$ and $\mathrm{Li}-\mathrm{P}$ coatings produced surfaces with rough morphologies, made of spheroidal formations (Figure 8), known in the literature as particulates. It should be stressed here that, their origin and dependence on the target composition represent common features of the PLD process [46].

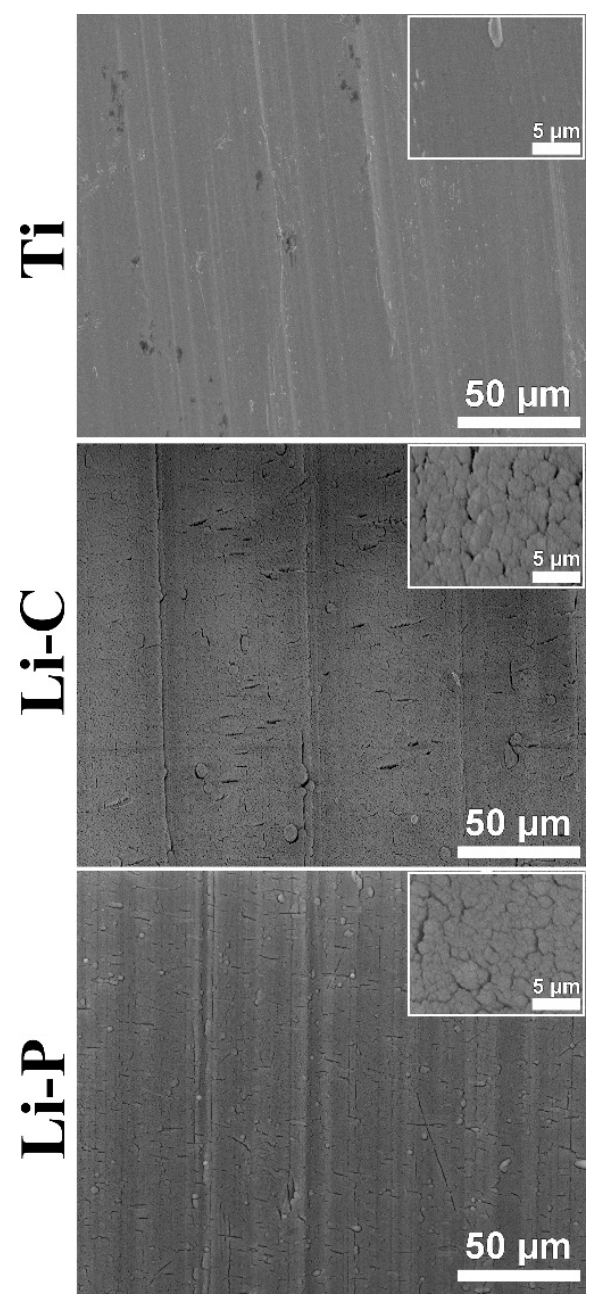

Figure 8. Scanning electron microscopy (SEM) micrographs of control and functionalized (with Li-C and Li-P coatings) 3D Ti implants before implantation. 
After the extraction procedure, SEM examinations of control and functionalized 3D Ti implants were carried out under two magnifications, i.e., 300× and 2000×, respectively (Figure 9).

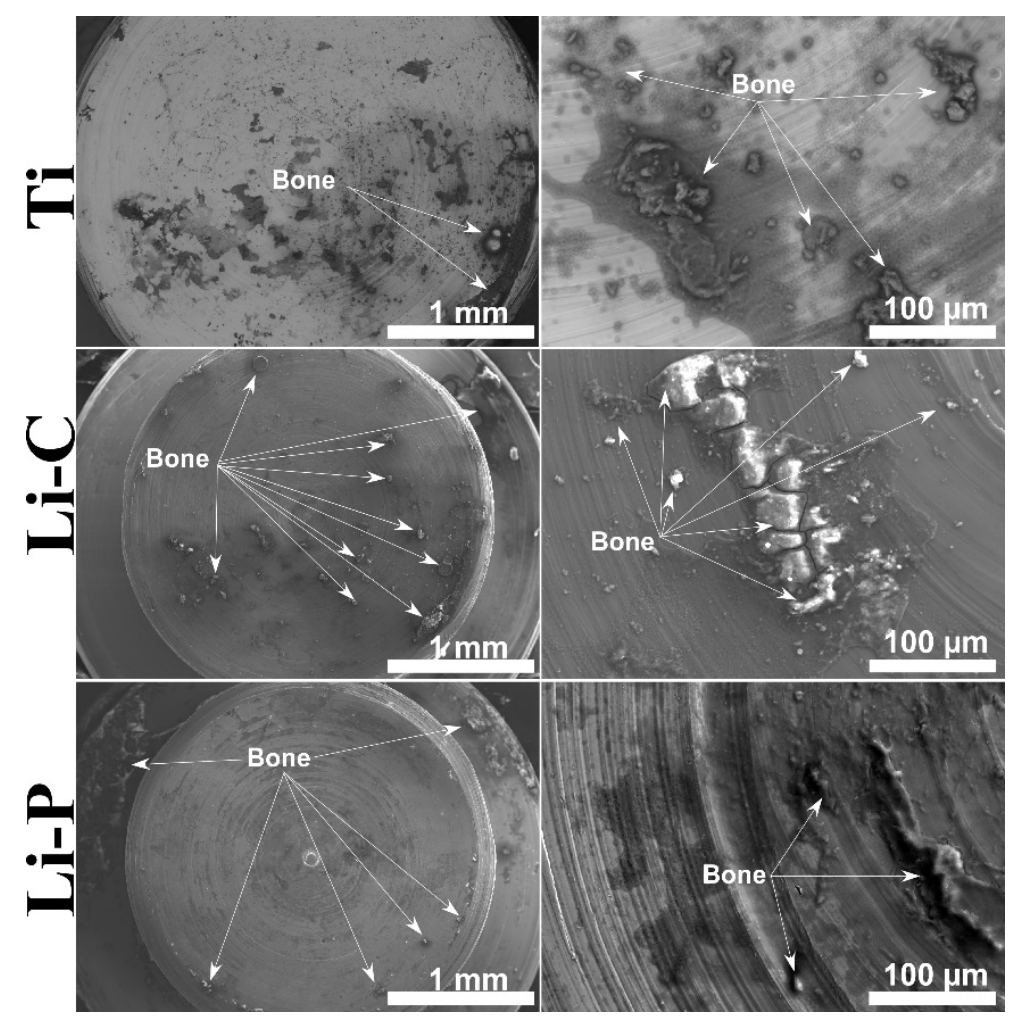

Figure 9. SEM micrographs indicating bone detachment on the surface of a control and functionalized (with Li-C and Li-P coatings) Ti implant, at 4 weeks after surgery.

The inferred adherence ratio of the remaining bone fragments onto the surface of the extracted implants revealed values of $2.69 \pm 0.03 \%$, in the case of control 3D Ti implants, and of $3.54 \pm 0.03 \%$ and $3.66 \pm 0.02 \%$, in the case of the implants functionalized with Li-P and Li-C coatings, respectively. This corresponds to adherence ratios up to $\sim 38 \%$ higher in the case of functionalized 3D Ti implants as compared to control ones (Figure 10). This could be indicative of an enhanced osseointegration process. Moreover, the higher values of the detachment force inferred in the case of functionalized 3D Ti implants, in comparison to control ones (presented in Figures 6 and 7), should support this observation. It is important to stress that, the presence of such osseous structures onto the surface of 3D Ti implants suggests, besides the beginning of the implant integration process into the bone, the absence of any adverse reactions at the implantation site. 


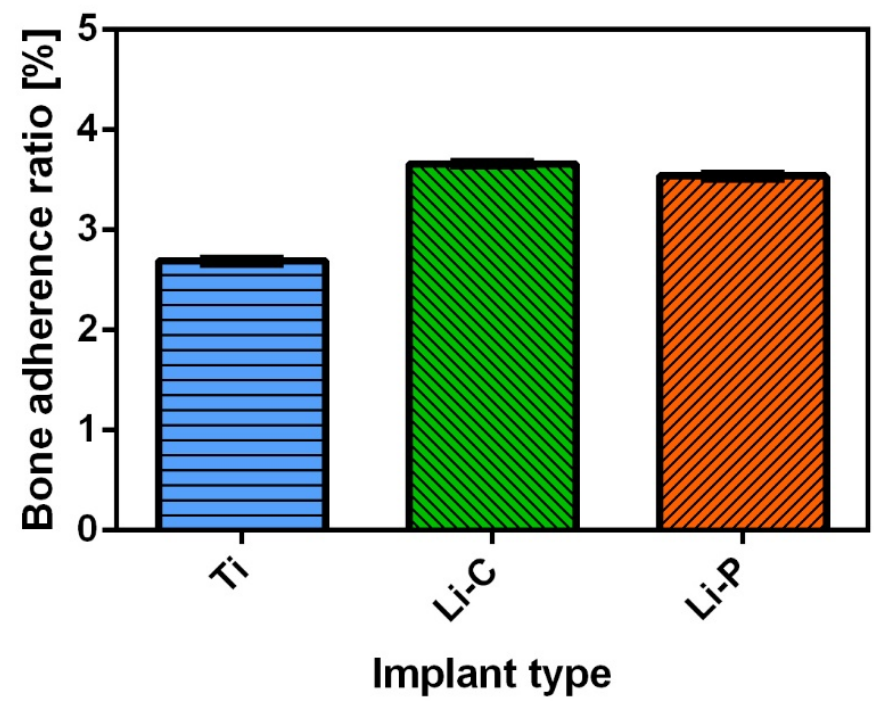

Figure 10. Inferred bone adherence ratio in the case of control and functionalized (with Li-C and Li-P coatings) 3D Ti implants $(n=3)$, at 4 weeks after surgery.

\section{Discussion}

The rabbit's bones manifest faster changes and turnover in comparison to larger animal models [47]. Taking into consideration the difficulty to extrapolate the results obtained on rabbit bones to the human ones, the current study represents a screening for implant design and a validation of the used biomaterials, before their testing on larger animal models.

Due to its attractive biological properties and resemblance to the mineral part of the bone, HA currently represents one of the most used CaPs. To overcome the drawback that synthetic HA does not completely match the chemical composition of bone mineral component [48], continuous efforts have been dedicated to find alternative, low-cost methods to produce HA, such as obtaining it from sustainable, biological (BioHA) resources (biogenic, mammalian, and/or natural fish bones). One should note that BioHA is a carbonated, non-stoichiometric Ca-deficient material, which is different from synthetic HA in terms of composition, stoichiometry, crystal size/morphology, crystallinity degree, degradation rate, and overall biological performance [29]. Another important aspect of the growing interest of using BioHA materials is related to the presence of trace elements and functional groups, which modify the chemical formula of the natural HA in bone. While the chemically-synthesized HA lacks these elements, mammalian bones contain a higher source of ions and trace elements [49-51], with $\mathrm{Na}^{+}$and $\mathrm{Mg}^{2+}$ as the most frequently found ones [29]. One notes that, the presence of these ions alongside HA plays an important role in the development of teeth and bone, whereas their absence could cause fragility or bone loss [6].

The three main steps for bone formation are (i) cellular osteogenic differentiation, (ii) matrix maturation and (iii) matrix mineralization. The initial adhesion of cells to an implant represents a key parameter for their proliferation and differentiation onto the surface of a biomaterial. The surface functionalization of implants by $\mathrm{CaP}$ coatings can ensure new bone formation, which might occur rapidly along the entire surface of the coating, as compared to the simple Ti implant (control) [52]. In this respect, in our previous studies [33,34], the effect of lithium addition $\left(\mathrm{Li}_{2} \mathrm{CO}_{3}\right.$ and/or $\left.\mathrm{Li}_{3} \mathrm{PO}_{4}\right)$ in BHA coatings synthesized by PLD was thoroughly examined from the physical-chemical, mechanical and cytocompatibility points of view. In comparison to bare Ti implants and simple (undoped) BHA coatings, Li-C and Li-P structures elicited improved wettability properties, which could further induce improved bone regeneration [33]. These characteristics seemed to have a beneficial influence on the overall cytotoxicity of the materials, the proliferation of human mesenchymal stem cells (hMSC) reaching its highest values in the case of Li-C coatings, followed by Li-P and simple (undoped) BHA ones. After $72 \mathrm{~h}$, the cellular viability of hMSC was superior for BHA-doped structures (Li-P and Li-C) 
as compared to simple (undoped) BHA ones. One should note that, the bonding strength values were two times (in the case of Li-P coatings) and up to more than three times (in the case of Li-C coatings) higher than the threshold ( $>15 \mathrm{MPa}$ ) imposed by the ISO standard regulating the load-bearing implant coatings [33]. The evaluation of simple (undoped) and doped (Li-P) BHA coatings to induce osteoblast (OB) cells differentiation was also performed [34]. The level of mineralization in the case of Li-P coatings was found to be higher and significantly higher as compared to simple (undoped) BHA ones and bare $\mathrm{Ti}$, respectively. In addition, when referring to the in situ level of alkaline phosphatase (which is an early marker of osteogenic differentiation), in hMSC and differentiated OB cells, the slightly improved results in the case of doped BHA (Li-P) structures in comparison to simple (undoped) BHA ones, could be explained by the characteristics of the coatings induced by the lithium doping agent (low roughness values and a hydrophilic behavior) [34]. Moreover, the comparative morphological investigations evidenced the presence of numerous nodules of mineralization originating from OB cells grown on the surface of Li-P coatings, as well as a network-like organization of OBs interconnected into the mineralized extracellular matrix. Therefore, it was suggested that this network of OBs could constitute the premise for in vitro early differentiation of cells and, consequently, for an improved osseointegration in vivo [34]. Analyzing the overall demonstrated improved characteristics of lithium-doped BHA structures in comparison to bare Ti implants and simple (undoped) BHA coatings, it was concluded that the incorporation of lithium might prove to be a delivery vehicle for bioactive agents to promote and even accelerate osseointegration in close relation with an improved anchorage of bone metallic implants. It should be emphasized here that, the current EU strategies applied for the optimization of in vivo experiments indicate that the maximum amount of relevant, high-quality data should be generated strictly in accordance with the $3 R$ s philosophy, i.e., reduction, refinement and replacement of animal use, as defined by Russell and Burch in 1959. Taking into consideration all these aspects, along with the fact that the aim of this pilot study was not to investigate the lithium effectiveness in in vivo experiments, but to demonstrate the superiority of lithium-doped BHA coatings over commercial Ti implants (which represent the "gold standard" for implantological applications), the use of simple (undoped) BHA coatings as a second control (next to bare Ti implants) was not considered necessary at this stage of research.

We have shown in the current study that, after implantation of the 3D printed coated and uncoated Ti implants, the bone in the vicinity of the devices started to react to the foreign body. In all cases, between 4 and 9 weeks the bone density increased. There was an obvious difference between the uncoated (control) 3D Ti implants and those functionalized with Li-C and Li-P coatings, in terms of bone density. The coated implants were always surrounded by a denser bone as compared to the uncoated ones, after 4 and 9 weeks, respectively. The denser bone around Li-C and Li-P coatings was expected, as the synthesized structures are based on natural HA, which is very similar to the mineral part of the bone. The material is biomimetic and bioactive, shielding the metallic implant and further stimulating the bone growth.

Apparently, between the Li-C and Li-P coated implants there were no obvious differences in terms of bone density. Therefore, to elucidate which of the two type of coatings could be more beneficial for the bone growth, in vivo mechanical tests were performed. It should be noted that, the optimal functioning of an endosseous implant depends on the mechanical stability of the biomaterial, which can be evaluated by extraction tests. To obtain information regarding the force that occurs between the bone tissue and implanted materials, various experimental study models have been developed, each of these approaches having its own particularities [44,47,53]. Most animal models currently used to study the retention of implants in bone are influenced by shear forces introduced during the retention tests. Push-out and/or torsion tests are generally used to analyze these forces. Push-out tests have some limitations regarding the evaluation of the bone-implant force. Therefore, the implant's shape, the degree of surface roughness and the good alignment between the implant clamping system and the pushing device can significantly influence test results. The use of the torsion test has the theoretical advantage of achieving an equal distribution of the force field at the bone-implant interface. 
On the other hand, these experiments are far more complicated and time-consuming as compared to the push-out ones. At the same time, the torsion test does not provide a clear distinction between the chemical bone-implant interaction and the mechanical retention because of the implant's surface roughness. Therefore, some studies, including the current one, have been concentrated on tensile strength measurements which, very important, can be influenced only by the chemical links between the implant and the bone [54-56]. Therefore, it is considered that by tensile tests the strength of the chemical bond can be measured directly. Thus, after 4 weeks of implantation, the value of the detachment force was higher in the case of 3D Ti implants functionalized with Li-C coatings in comparison to uncoated (control) ones. For the case of Li-P structures, this trend was similar, but there were particular instances when the results for coated and uncoated implants were very similar. The trend of higher detachment force was maintained for the case of 3D Ti implants functionalized with Li-P coatings as compared to the uncoated implants, however the effect seemed to be attenuated in respect to the Li-C coatings. After 9 weeks of implantation, it was observed that the detachment force was clearly superior ( 1.3 times) for the 3D Ti implants functionalized with Li-C coatings as compared to the ones functionalized with $\mathrm{Li}-\mathrm{P}(106.6 \pm 2.9 \mathrm{~N}$ vs. $83.7 \pm 2.1 \mathrm{~N})$. A possible explanation for the higher values of the bone strength in the first case (Li-C coatings), could be connected to an increased crystallinity of the structures [57]. In this respect, it was shown that the Li-C coatings displayed a higher degree of crystallinity as compared to Li-P ones [33].

In the case of biological HA materials, as compared to synthetic HA, the osseoconduction rate is faster because the latter (i) has a higher solubility (which increases if its chemical structure contains carbonate groups), and (ii) contains $\mathrm{Mg}^{2+}$ and $\mathrm{Na}^{+}$ions, which are two elements associated to bone remodeling [58]. This might represent one of the possible mechanisms through which the Li-C and $\mathrm{Li}-\mathrm{P}$ coatings can improve the process of osseoconduction. One should therefore emphasize on the increased values of the detachment force obtained at 9 weeks, which demonstrate the capacity for long-term attachment to bone of our functionalized 3D metallic implants.

Usually, lithium is incorporated into CaPs in form of $\mathrm{Li}_{2} \mathrm{O}[59,60]$. In the current study, we selected to mix it with $\mathrm{HA}$ in form of $\mathrm{Li}_{2} \mathrm{CO}_{3}$ and $\mathrm{Li}_{3} \mathrm{PO}_{4}$. The reason was that in an initial parametric study (data not shown here), $\mathrm{Li}_{2} \mathrm{O}$ mixed with natural origin $\mathrm{HA}$ displayed inferior results in vitro as compared to the $\mathrm{Li}_{2} \mathrm{CO}_{3}$ and $\mathrm{Li}_{3} \mathrm{PO}_{4}$ mixes. There were studies in the literature devoted to lithium relation to the human bone, some associating it with osteoporosis, while others signaling bone enhancement. In a study of Zamani et al. [61], it was shown that the treatment of patients with $\mathrm{Li}_{2} \mathrm{CO}_{3}$ preserved and even enhanced bone mass. Moreover, the results reported by Cohen et al. [62], demonstrated that $\mathrm{Li}_{2} \mathrm{CO}_{3}$ incorporated in drugs does not induce a risk of osteoporosis. One should note that, Li-P was tested in the current in vivo preliminary study because there are no reports in the dedicated literature related to its action on the bone. The results of the current work demonstrated that it possesses bioactive characteristics in conjunction with biological origin HA, however it was inferior to its Li-C counterpart.

All in all, the results of this preliminary in vivo assessment of the pulsed laser deposited BioHA doped with Li-C and Li-P coatings hold promise. Further and more insightful documentation on both the ingrowth characteristics' influence on the mechanical stability over various implantation time periods, and biomolecular analyses (i.e., related to gene expression of osteoblasts in contact with bone substitutes and/or HA [63]), will be considered. In addition, detailed in vivo studies, in which simple (undoped) BHA coatings will be used as controls, will be imagined not only to assess the lithium effectiveness in in vivo experiments, but to demonstrate their superiority also over the commercial Ti implants. All these investigations are necessary and should stand as the subject of a dedicated study which should start, however, from the results of this preliminary work.

\section{Conclusions}

Pulsed laser deposition was used for the synthesis of biological-derived hydroxyapatite doped with lithium carbonate (Li-C) and phosphate ( $\mathrm{Li}-\mathrm{P})$ coatings. The $\mathrm{Li}-\mathrm{C}$ and $\mathrm{Li}-\mathrm{P}$ structures were 
investigated in vivo, as coatings onto 3D metallic implants which were inserted in rabbits' femoral condyles, for 4 and 9 weeks, respectively. The bone density measurements of the functionalized Ti implants, performed either at 4 or 9 weeks, showed superior values in comparison to simple (control) Ti ones. The inferred detachment force values of the functionalized Ti implants were $\sim 2$ times higher than those registered for the corresponding control ones. When referring to longer implantation time periods (i.e., 9 weeks), the extraction test results indicated improved bonding strength values ( $\sim 5$ times higher) of the functionalized Ti implants as compared to the same structures, but corresponding to an implantation time period of 4 weeks. Therefore, the mechanical testing is indicated as a promising tool to investigate the early phase of 3D Ti implants attachment to bone.

The demonstrated improvement of in vivo mechanical characteristics of the $\mathrm{Li}-\mathrm{C}$ and Li-P synthesized coatings (as compared to control, commercial Ti implants), along with the fact that the materials used in this study were fabricated from natural, renewable sources, could stand for a pioneering contribution to the progress of medical devices. These important aspects should be taken into consideration when advancing this type of implant modification as an adequate choice to bare, non-deposited Ti implants for the manufacturing of a new generation of orthopedic implants, which will integrate better and much faster into the living body, corroborated with a substantially improved life-time.

\section{Patents}

A patent application entitled "Additive manufacturing of fixing devices for metallic implants by laser melting deposition method", by Chioibasu Diana, Mihai Sabin-Andrei, Duta Liviu-Marian, Popescu C. Andrei, containing information related to the procedure and devices used for fixing the 3D metallic implants both during the PLD experiments and during the post deposition thermal treatments, was submitted for evaluation to State Office for Inventions and Trademarks (OSIM), A00214/22.04.2020.

Author Contributions: Conceptualization, L.D.; methodology, L.D., O.A.Z., G.P.-P., D.C., and F.N.O.; software, L.D., J.N., R.P.M., O.A.Z., G.P.-P., D.C., and A.C.P.; validation, L.D., J.N., R.P.M. and A.C.P.; formal analysis, L.D.; investigation, J.N., R.P.M., and O.A.Z.; resources, L.D., F.N.O., and A.C.P.; data curation, L.D. and J.N.; writing—original draft preparation, L.D.; writing—review and editing, L.D., J.N., R.M., O.A.Z., G.P.-P, D.C., F.N.O., and A.C.P.; visualization, L.D., J.N., and A.C.P.; supervision, L.D.; project administration, L.D. and F.N.O.; funding acquisition, L.D. and A.C.P. All authors have read and agreed to the published version of the manuscript.

Funding: L.D. acknowledges the support from a grant of Ministry of Research and Innovation, CNCS-UEFISCDI, No. PN-III-P1-1.1-PD-2016-1568 (PD 6/2018), within PNCDI III. A.C.P. and D.C. have received funding from the PN-III-P1-1.1-TE-2016-2015 (TE136/2018) Project. This work was also supported by the Romanian Ministry of Education and Research, under Romanian National Nucleus Program LAPLAS VI-Contract 16N/2019.

Acknowledgments: All authors acknowledge George E. Stan for his useful comments and kind help.

Conflicts of Interest: The authors declare no conflict of interest.

\section{References}

1. Popa, A.C.; Stan, G.E.; Besleaga, C.; Ion, L.; Maraloiu, V.A.; Tulyaganov, D.U.; Ferreira, J.M.F. Submicrometer Hollow Bioglass Cones Deposited by Radio Frequency Magnetron Sputtering: Formation Mechanism, Properties, and Prospective Biomedical Applications. ACS Appl. Mater. Interfaces 2016, 8, 4357-4367. [CrossRef] [PubMed]

2. Stan, G.E.; Popescu, A.C.; Mihailescu, I.N.; Marcov, D.A.; Mustata, R.C.; Sima, L.E.; Petrescu, S.M.; Ianculescu, A.; Trusca, R.; Morosanu, C.O. On the bioactivity of adherent bioglass thin films synthesized by magnetron sputtering techniques. Thin Solid Films 2010, 518, 5955-5964. [CrossRef]

3. Šupová, M. Substituted hydroxyapatites for biomedical applications: A review. Ceram. Int. 2015, 41, 9203-9231. [CrossRef]

4. Graziani, G.; Boi, M.; Bianchi, M. A Review on Ionic Substitutions in Hydroxyapatite Thin Films: Towards Complete Biomimetism. Coatings 2018, 8, 269. [CrossRef]

5. Oladele, I.O.; Agbabiaka, O.; Olasunkanmi, O.G.; Balogun, A.O.; Popoola, M.O. Non-synthetic sources for the development of hydroxyapatite. J. Appl. Biotechnol. Bioeng. 2018, 5, 92-99. [CrossRef] 
6. Akram, M.; Ahmed, R.; Shakir, I.; Ibrahim, W.A.W.; Hussain, R. Extracting hydroxyapatite and its precursors from natural resources. J. Mater. Sci. 2014, 49, 1461-1475. [CrossRef]

7. Tite, T.; Popa, A.C.; Balescu, L.M.; Bogdan, I.M.; Pasuk, I.; Ferreira, J.M.F.; Stan, G.E. Cationic substitutions in hydroxyapatite: Current status of the derived biofunctional effects and their in vitro interrogation methods. Materials 2018, 11, 2081. [CrossRef]

8. Ballini, A.; Mastrangelo, F.; Gastaldi, G.; Tettamanti, L.; Bukvic, N.; Cantore, S.; Cocco, T.; Saini, R.; Desiate, A.; Gherlone, E.; et al. Osteogenic differentiation and gene expression of dental pulp stem cells under low-level laser irradiation: A good promise for tissue engineering. J. Biol. Regul. Homeost. Agents 2015, 29, 813-822. [PubMed]

9. Bose, S.; Tarafder, S.; Bandyopadhyay, A. Hydroxyapatite coatings for metallic implants. In Hydroxyapatite (Hap) for Biomedical Applications; Mucalo, M., Ed.; Woodhead Publishing Series in Biomaterials; Elsevier: Amsterdam, The Netherlands, 2015; pp. 143-157. [CrossRef]

10. Barrere, F.; van der Valk, C.M.; Meijer, G.; Dalmeijer, R.A.; Groot, K.; Layrolle, P. Osteointegration of biomimetic apatite coating applied onto dense and porous metal implants in femurs of goats. J. Biomed. Mater. Res. B Appl. Biomater. 2003, 67, 655-665. [CrossRef]

11. Surmenev, R.A.; Surmeneva, M.A. A critical review of decades of research on calcium phosphate-based coatings: How far are we from their widespread clinical application? Curr. Opin. Biomed. Eng. 2019, 10, 35-44. [CrossRef]

12. Dorozhkin, S.V. Biphasic, triphasic, and multiphasic calcium orthophosphates. In Advanced Ceramic Materials; Tiwari, A., Gerhardt, R.A., Szutkowska, M., Eds.; Wiley, Scrivener Publishing: Austin, TX, USA, 2016; pp. 33-95. [CrossRef]

13. Crespi, R.; Capparè, P.; Gherlone, E. Comparison of magnesium-enriched hydroxyapatite and porcine bone in human extraction socket healing: A histologic and histomorphometric evaluation. Int. J. Oral. Maxillofac. Implant. 2011, 26, 1057-1062. [PubMed]

14. Crespi, R.; Capparè, P.; Gherlone, E. Magnesium-enriched hydroxyapatite compared to calcium sulfate in the healing of human extraction sockets: Radiographic and histomorphometric evaluation at 3 months. J. Periodontol. 2009, 80, 210-218. [CrossRef] [PubMed]

15. Gherlone, E.F.; Capparé, P.; Tecco, S.; Polizzi, E.; Pantaleo, G.; Gastaldi, G.; Grusovin, M.G. A Prospective Longitudinal Study on Implant Prosthetic Rehabilitation in Controlled HIV-Positive Patients with 1-Year Follow-Up: The Role of CD4+ Level, Smoking Habits, and Oral Hygiene. Clin. Implant Dent. Relat. Res. 2016, 18, 955-964. [CrossRef] [PubMed]

16. Pesce, V.; Speciale, D.; Sammarco, G.; Patella, S.; Spinarelli, A.; Patella, V. Surgical approach to bone healing in osteoporosis. Clin. Cases Miner. Bone Metab. 2009, 6, 131-135. [PubMed]

17. León, B.; John, J. Thin Calcium Phosphate Coatings for Medical Implants; Springer-Verlag: New York, NY, USA, 2009; pp. 1-328. [CrossRef]

18. Available online: https://www.eos.info/en/3d-printing-examples-applications/people-health/medical-3dprinting (accessed on 9 October 2020).

19. Jiaxiang, Z.; Anh, Q.V.; Xin, F.; Suresh, B.; Michael, A.R. Pharmaceutical Additive Manufacturing: A Novel Tool for Complex and Personalized Drug Delivery Systems. AAPS PharmSciTech 2018, 19, 3388-3402. [CrossRef]

20. Sima, L.E.; Stan, G.E.; Morosanu, C.O.; Melinescu, A.; Ianculescu, A.; Melinte, R.; Neamtu, J.; Petrescu, S.M. Differentiation of mesenchymal stem cells onto highly adherent radio frequency-sputtered carbonated hydroxylapatite thin films. J. Biomed. Mater. Res. A 2010, 95, 1203-1214. [CrossRef]

21. Visan, A.; Grossin, D.; Stefan, N.; Duta, L.; Miroiu, F.M.; Stan, G.E.; Sopronyi, M.; Luculescu, C.; Freche, M.; Marsan, O.; et al. Biomimetic nanocrystalline apatite coatings synthesized by Matrix Assisted Pulsed Laser Evaporation for medical applications. Mater. Sci. Eng. B Adv. 2014, 181, 56-63. [CrossRef]

22. Graziani, G.; Bianchi, M.; Sassoni, E.; Russo, A.; Marcacci, M. Ion-substituted calcium phosphate coatings deposited by plasma-assisted techniques: A review. Mater. Sci. Eng. C 2017, 74, 219-229. [CrossRef]

23. Vranceanu, D.M.; Ionescu, I.C.; Ungureanu, E.; Cojocaru, M.O.; Vladescu, A.; Cotrut, C.M. Magnesium Doped Hydroxyapatite-Based Coatings Obtained by Pulsed Galvanostatic Electrochemical Deposition with Adjustable Electrochemical Behavior. Coatings 2020, 10, 727. [CrossRef] 
24. Stewart, C.; Akhavan, B.; Wise, S.G.; Bilek, M.M.M. A review of biomimetic surface functionalization for bone integrating orthopedic implants: Mechanisms, current approaches, and future directions. Prog. Mater. Sci. 2019, 106, 100588. [CrossRef]

25. Asri, R.I.; Harun, W.S.; Hassan, M.A.; Ghani, S.A.; Buyong, Z. A review of hydroxyapatite-based coating techniques: Sol-gel and electrochemical depositions on biocompatible metals. J. Mech. Behav. Biomed. Mater. 2016, 57, 95-108. [CrossRef] [PubMed]

26. Chen, N.; Kim, D.H.; Kovacik, P.; Sojoudi, H.; Wang, M.; Gleason, K.K. Polymer thin films and surface modification by chemical vapor deposition: Recent progress. Annu. Rev. Chem. Biomol. Eng. 2016, 7, 373-393. [CrossRef]

27. Kumar, R.; Kumar, G.; Umar, A. Pulse laser deposited nanostructured ZnO thin films: A review. J. Nanosci. Nanotechnol. 2014, 14, 1911-1930. [CrossRef] [PubMed]

28. Chen, L.; Komasa, S.; Hashimoto, Y.; Hontsu, S.; Okazaki, J. In Vitro and In Vivo Osteogenic Activity of Titanium Implants Coated by Pulsed Laser Deposition with a Thin Film of Fluoridated Hydroxyapatite. Int. J. Mol. Sci. 2018, 19, 1127. [CrossRef] [PubMed]

29. Duta, L.; Popescu, A.C. Current Status on Pulsed Laser Deposition of Coatings from Animal-Origin Calcium Phosphate Sources. Coatings 2019, 9, 335. [CrossRef]

30. Bao, Q.; Chen, C.; Wang, D.; Ji, Q.; Lei, T. Pulsed laser deposition and its current research status in preparing hydroxyapatite thin films. Appl. Surf. Sci. 2005, 252, 1538-1544. [CrossRef]

31. Kuzanyan, A.S.; Kuzanyan, A.A. Pulsed Laser Deposition of Large-Area Thin Films and Coatings. In Applications of Laser Ablation-Thin Film Deposition, Nanomaterial Synthesis and Surface Modification; Yang, D., Ed.; InTech: Rijeka, Croatia, 2016. [CrossRef]

32. Greer, J.A. History and current status of commercial pulsed laser deposition equipment. J. Phys. D Appl. Phys. 2014, 47, 034005. [CrossRef]

33. Popescu, A.C.; Florian, P.E.; Stan, G.E.; Popescu-Pelin, G.; Zgura, I.; Enculescu, M.; Oktar, F.N.; Trusca, R.; Sima, L.E.; Roseanu, A.; et al. Physical-chemical characterization and biological assessment of simple and lithium-doped biological-derived hydroxyapatite thin films for a new generation of metallic implants. Appl. Surf. Sci. 2018, 439, 724-735. [CrossRef]

34. Florian, P.E.; Duta, L.; Grumezescu, V.; Popescu-Pelin, G.; Popescu, A.C.; Oktar, F.N.; Evans, R.W.; Constantinescu, A.R. Lithium-Doped Biological-Derived Hydroxyapatite Coatings Sustain In Vitro Differentiation of Human Primary Mesenchymal Stem Cells to Osteoblasts. Coatings 2019, 9, 781. [CrossRef]

35. Duta, L.; Chifiriuc, M.C.; Popescu-Pelin, G.; Bleotu, C.; Gradisteanu, G.P.; Anastasescu, M.; Achim, A.; Popescu, A. Pulsed Laser Deposited Biocompatible Lithium-Doped Hydroxyapatite Coatings with Antimicrobial Activity. Coatings 2019, 9, 54. [CrossRef]

36. Duta, L.; Oktar, F.N.; Stan, G.E.; Popescu-Pelin, G.; Serban, N.; Luculescu, C.; Mihailescu, I.N. Novel doped hydroxyapatite thin films obtained by pulsed laser deposition. Appl. Surf. Sci. 2013, 265, 41-49. [CrossRef]

37. Duta, L.; Mihailescu, N.; Popescu, A.C.; Luculescu, C.R.; Mihailescu, I.N.; Cetin, G.; Gunduz, O.; Oktar, F.N.; Popa, A.C.; Kuncser, A.; et al. Comparative physical, chemical and biological assessment of simple and titanium-doped ovine dentine-derived hydroxyapatite coatings fabricated by pulsed laser deposition. App Surf. Sci. 2017, 413, 129-139. [CrossRef]

38. Eisenhart, S. EU Regulation 722. In New EU Animal Tissue Regulations in Effect for Some Medical Devices; Emergo: Hong Kong, China, 2013; Available online: https://www.emergobyul.com/blog/2013/09/new-euanimaltissueregulations-effect-some-medical-devices (accessed on 11 September 2020).

39. ISO 22442-1. Medical Devices Utilizing Animal Tissues and Their Derivatives-Part 1: Application of Risk Management; International Organization for Standardization: Berlin, Germany, 2015.

40. Chioibasu, D.; Mihai, S.-A.; Duta, L.; Popescu, A.C. Additive Manufacturing of Fixing Devices for Metallic Implants by “Laser Melting Deposition” Method. status (unpublished Patent application, A00214, submitted to State Office for Inventions and Trademarks, OSIM, 22 April 2020).

41. Lu, Y.P.; Chen, Y.M.; Li, S.T.; Wang, J.H. Surface nanocrystallization of hydroxyapatite coating. Acta Biomater. 2008, 4, 1865-1872. [CrossRef] [PubMed]

42. Neyt, J.G.; Buckwalter, J.A.; Carroll, N.C. Use of animal models in musculoskeletal research. Iowa Orthop. J. 1998, 18, 118-123. 
43. Gilsanz, V.; Roe, T.F.; Gibbens, D.T.; Schulz, E.E.; Carlson, M.E.; Gonzalez, O.; Boechat, M.I. Effect of sex steroids on peak bone density of growing rabbits. Am. J. Physiol. 1988, 255, E416-E421. [CrossRef]

44. Salou, L.; Hoornaert, A.; Louarn, G.; Layrolle, P. Enhanced osseointegration of titanium implants with nanostructured surfaces: An experimental study in rabbits. Acta Biomater. 2015, 11, 494-502. [CrossRef] [PubMed]

45. Yan, J.; Sun, J.-F.; Chu, P.K.; Han, Y.; Zhang, Y.-M. Bone integration capability of a series of strontium-containing hydroxyapatite coatings formed by micro-arc oxidation. J. Biomed. Mater. Res. A 2013, 101, 2465-2480. [CrossRef]

46. Eason, R. Pulsed Laser Deposition of Thin FILMS: Applications-Led Growth of Functional Materials, 1st ed.; Wiley \& Sons Interscience: Hoboken, NY, USA, 2007; pp. 1-705.

47. Pearce, A.I.; Richards, R.G.; Milz, S.; Schneider, E.; Pearce, S.G. Animal models for implant biomaterial research in bone: A review. Eur. Cells Mater. 2007, 13, 1-10. [CrossRef] [PubMed]

48. Goller, G.; Oktar, F.N.; Agathopoulos, S.; Tulyaganov, D.U.; Ferreira, J.M.F.; Kayali, E.S.; Peker, I. Effect of sintering temperature on mechanical and microstructural properties of bovine hydroxyapatite (BHA). J. Sol-Gel Sci. Techn. 2006, 37, 111-115. [CrossRef]

49. Sun, R.-X.; Lv, Y.; Niu, Y.-R.; Zhao, X.-H.; Cao, D.-S.; Tang, J.; Sun, X.-C.; Chen, K.-Z. Physicochemical and biological properties of bovine-derived porous hydroxyapatite/collagen composite and its hydroxyapatite powders. Ceram. Int. 2017, 43, 16792-16798. [CrossRef]

50. Rahavi, S.S.; Ghaderi, O.; Monshi, A.; Fathi, M.H. A comparative study on physicochemical properties of hydroxyapatite powders derived from natural and synthetic sources. Russ. J. Non-Ferrous Metals 2017, 58, 276-286. [CrossRef]

51. Ofudje, E.A.; Rajendran, A.; Adeogun, A.I.; Idowu, M.A.; Kareem, S.O.; Pattanayak, D.K. Synthesis of organic derived hydroxyapatite scaffold from pig bone waste for tissue engineering applications. Adv. Powder Technol. 2017, 29, 1-8. [CrossRef]

52. Su, Y.; Cockerill, I.; Zheng, Y.; Tang, L.; Qin, Y.-X.; Zhu, D. Biofunctionalization of metallic implants by calcium phosphate coatings. Bioact. Mater. 2019, 4, 196-206. [CrossRef]

53. Sul, Y.-T.; Johansson, C.; Albrektsson, T. A novel in vivo method for quantifying the interfacial biochemical bond strength of bone implants. J. R Soc. Interface 2009, 7, 81-90. [CrossRef] [PubMed]

54. Shannon, F.J.; Cottrell, J.M.; Deng, X.-H.; Crowder, K.N.; Doty, S.B.; Avaltroni, M.J.; Warren, R.F.; Wright, T.M.; Schwartz, J. A novel surface treatment for porous metallic implants that improves the rate of bony ongrowth. J. Biomed. Mater. Res. A 2008, 86, 857-864. [CrossRef]

55. Schumacher, T.C.; Tushtev, K.; Wagner, U.; Becker, C.; Große Holthaus, M.; Hein, S.B.; Haack, J.; Engelhardt, C.H.M.; Khassawna, T.E.; Rezwan, K. A novel, hydroxyapatite-based screw-like device for anterior cruciate ligament (ACL) reconstructions. Knee 2017, 24, 933-939. [CrossRef]

56. Aparicioa, C.; Padrósb, A.; Gil, F.-J. In vivo evaluation of micro-rough and bioactive titanium dental implants using histometry and pull-out tests. J. Mech. Behav. Biomed. 2011, 4, 1672-1682. [CrossRef]

57. Kumar, R.R.; Maruno, S. Functionally graded coatings of HA-G-Ti composites and their in vivo studies. Mater. Sci. Eng. A Struct. 2002, 334, 156-162. [CrossRef]

58. Hayami, T.; Hontsu, S.; Higuchi, Y.; Nishikawa, H.; Kusunoki, M. Osteoconduction of a stoichiometric and bovine hydroxyapatite bilayer-coated implant, hydroxyapatite bilayer-coated implant. Clin. Oral Implant. Res. 2011, 22, 774-776. [CrossRef]

59. Zhang, K.; Alaohali, A.; Sawangboon, N.; Sharpe, P.T.; Brauer, D.S.; Gentleman, E. A comparison of lithium-substituted phosphate and borate bioactive glasses for mineralised tissue repair. Dent. Mater. 2019, 35, 919-927. [CrossRef]

60. Khan, P.K.; Mahato, A.; Kundu, B.; Nandi, S.K.; Mukherjee, P.; Datta, S.; Sarkar, S.; Mukherjee, J.; Nath, S.; Balla, V.K.; et al. Influence of single and binary doping of strontium and lithium on in vivo biological properties of bioactive glass scaffolds. Sci. Rep. UK 2016, 6, 32964. [CrossRef]

61. Zamani, A.; Omrani, G.R.; Nasab, M.M. Lithium's effect on bone mineral density. Bone 2009, 44, 331-334. [CrossRef] 
62. Cohen, O.; Rais, T.; Lepkifker, E.; Vered, I. Lithium Carbonate Therapy is not a Risk Factor for Osteoporosis. Horm. Metab. Res. 1998, 30, 594-597. [CrossRef] [PubMed]

63. Crespi, R.; Capparé, P.; Romanos, G.E.; Mariani, E.; Benasciutti, E.; Gherlone, E. Corticocancellous porcine bone in the healing of human extraction sockets: Combining histomorphometry with osteoblast gene expression profiles in vivo. Int. J. Oral Maxillofac. Implant. 2011, 26, 866-872. [PubMed]

Publisher's Note: MDPI stays neutral with regard to jurisdictional claims in published maps and institutional affiliations.

(C) 2020 by the authors. Licensee MDPI, Basel, Switzerland. This article is an open access article distributed under the terms and conditions of the Creative Commons Attribution (CC BY) license (http://creativecommons.org/licenses/by/4.0/). 